\title{
Variabilidade espacial do risco de contaminação de águas subterrâneas por tebuthiuron em área de cana-de-açúcar
}

\author{
Diego Augusto de Campos Moraes ${ }^{(1)}$, Claudio Aparecido Spadotto(2), \\ Anderson Antônio da Conceição Sartori ${ }^{(3)}$ e Célia Regina Lopes Zimback ${ }^{(1)}$
}

\begin{abstract}
(1)Universidade Estadual Paulista, Faculdade de Ciências Agronômicas, Rua José Barbosa de Barros, no 1.780, CEP 18610-307 Botucatu, SP, Brasil. E-mail: die.gomoraes45@gmail.com, czimback@gmail.com (2)Embrapa Gestão Territorial, Avenida Soldado Passarinho, no 303, Fazenda Chapadão, CEP 13070-115 Campinas, SP, Brasil. E-mail: claudio.spadotto@embrapa.br (3)Universidade do Sagrado Coração, Rua Irmã Arminda, no 10-50, Jardim Brasil, CEP 17011-160 Bauru, SP, Brasil. E-mail: sartori80@gmail.com
\end{abstract}

Resumo - O objetivo deste trabalho foi avaliar o risco de contaminação da água subterrânea por tebuthiuron, por meio da modelagem espacial, em área de cultivo de cana-de-açúcar, no Município de São Manuel, no Estado de São Paulo. Utilizou-se o modelo Araquá, para gerar estimativas pontuais da concentração, tendo-se considerado simulações no tocante às profundidades de 100 e $200 \mathrm{~cm}$ do lençol freático e lâminas de irrigação de 0 e $2.500 \mathrm{~mm}$. Como as estimativas de concentração de tebuthiuron não apresentaram dependência espacial, os mapas temáticos de risco de contaminação de águas subterrâneas foram obtidos pelo método de interpolação pelo inverso da distância. Ao se considerar todas as simulações, conclui-se que não haveria contaminação da água subterrânea por tebuthiuron, nas duas profundidades do lençol freático, com ou sem irrigação. A interpolação das estimativas das concentrações de tebuthiuron na água subterrânea proporciona a espacialização e a rápida visualização das áreas de maior risco de contaminação.

Termos para indexação: geoestatística, herbicida, lençol freático, mapas de risco, simulação.

\section{Spatial variability of groundwater contamination risk by tebuthiuron in sugarcane area}

\begin{abstract}
The objective of this work was to evaluate the groundwater contamination risk by tebuthiuron in a sugarcane area, through spatial modeling, in the municipality of São Manuel, in the state of São Paulo, Brazil. The Araquá model was used to generate specific concentration estimates, considering simulations regarding different water table depths $(100$ and $200 \mathrm{~cm})$ and irrigation depths $(0$ and 2,500 mm). As the estimated concentrations of tebuthiuron did not show spatial dependence, the thematic maps of risk of groundwater contamination were obtained through the method of interpolation by the inverse distance. Considering all the simulations, it can be concluded that there would be no groundwater contamination by tebuthiuron at the two depths of the water table, with or without irrigation. The interpolation of the estimated concentrations of tebuthiuron in groundwater provides the spatialization and a quick view of the areas with higher contamination risk.
\end{abstract}

Index terms: geostatistics, herbicide, water table, risk maps, simulation.

\section{Introdução}

O cultivo da cana-de-açúcar é uma das mais importantes atividades econômicas do Brasil em virtude da sua alta eficiência e competitividade, resultando em produtos como açúcar, álcool, combustíveis e até mesmo uso do bagaço para geração de energia (Silveira et al., 2014).

Um dos problemas de seu cultivo, no Brasil, está relacionado com a interferência das plantas daninhas, para as quais o método empregado para o controle é a aplicação de herbicidas (Silva et al., 2015). O tebuthiuron é um herbicida residual amplamente utilizado nos canaviais, no sistema tradicional, em aplicações de pré-emergência, para controle das principais espécies anuais infestantes da cultura (Tofoli et al., 2009).

No entanto, os defensivos agrícolas podem provocar efeitos prejudiciais ao meio ambiente, além de representar perigo aos seres humanos. O tebuthiuron, além de alta solubilidade em água, apresenta alta persistência em virtude da sua elevada meia-vida e da baixa adsorção às partículas do solo e matéria orgânica, 
o que facilita seu movimento até os compartimentos aquáticos (Franco-Bernardes et al., 2014). Em relação à toxicidade a peixes e outros organismos aquáticos, o tebuthiuron é praticamente não tóxico (Estados Unidos, 2013). No entanto, os efeitos tóxicos sobre esses organismos são poucos conhecidos, necessitando de mais estudos.

Modelos matemáticos implementados em simuladores foram desenvolvidos e empregados nos estudos do comportamento ambiental de defensivos agrícolas, como CMLS (Chemical Movement in Layered Soils) (Nofziger \& Hornsby, 1987) e Pearl (Tiktak et al., 2000). O software Araquá (Spadotto et al., 2010) foi desenvolvido para estimar a concentração pontual de um defensivo agrícola em águas subterrâneas e superficiais em condições brasileiras de clima e solo.

O movimento de outros herbicidas no solo, como atrazina, também foi avaliado em simulações com os modelos CMLS, Pearl e Macro (Cerdeira et al., 2005; Scorza Júnior \& Silva, 2008; Giannouli \& Antonopoulos, 2015). Recentemente, Pullan et al. (2016) desenvolveram e aplicaram um novo modelo do destino e transporte de defensivos agrícolas para uso em avaliação de risco ambiental, considerando a potabilidade da água.

Complementarmente, a espacialização das concentrações de defensivos agrícolas estimadas nos compartimentos aquáticos, pelos simuladores, pode fornecer subsídios à tomada de decisão em relação à contaminação dos recursos hídricos. Akbar e Lin (2010) desenvolveram o ArcPRZM-3, o qual é a integração do simulador PRZM versão 3 com SIG (Sistema de Informações Geográficas), para simular o potencial de lixiviação do herbicida bentazona no solo até águas subterrâneas. Moraes et al. (2012) obtiveram a variabilidade espacial das estimativas de concentração do tebuthiuron na água subterrânea utilizando o software Araquá e o método de interpolação por krigagem ordinária. Nota-se que poucos trabalhos consideraram o defensivo tebuthiuron e a espacialização de suas estimativas de concentração na água subterrânea.

O objetivo deste trabalho foi avaliar o risco de contaminação da água subterrânea por tebuthiuron, por meio da modelagem espacial, em área de cultivo de cana-de-açúcar, no Município de São Manuel, no Estado de São Paulo.

\section{Material e Métodos}

O estudo foi conduzido em área próxima ao Município de São Manuel, SP, entre as coordenadas de $22^{\circ} 45^{\prime}$ de latitude sul e $48^{\circ} 30^{\prime}$ de longitude a oeste de Greenwich. A altitude média é de $520 \mathrm{~m}$, com as temperaturas médias mínimas e máximas no verão de 19 e $29^{\circ} \mathrm{C}$, e no inverno de 12 e $25^{\circ} \mathrm{C}$, respectivamente. O tipo climático predominante na região é o Cwa, de acordo com a classificação de Köeppen, caracterizado como clima tropical de altitude, com inverno seco e verão quente.

A precipitação anual na área de estudo é de 1.507,23 mm (Agritempo, 2014), e o dado de evapotranspiração anual é de 1.107,00 mm (Ciiagro, 2014). O corpo de água subterrâneo considerado no trabalho foi o lençol freático, sendo simuladas duas profundidades desde a superfície do solo: $100 \mathrm{~cm}$ e $200 \mathrm{~cm}$. Foram consideradas as situações sem irrigação e com uma lâmina de irrigação anual simulada de $2.500 \mathrm{~mm}$.

O solo da área de estudo é classificado como Latossolo Vermelho distroférrico (Claessen, 1997), textura arenosa, consistência úmida friável. A grade amostral foi definida com intervalos regulares de $100 \mathrm{~m}$, ou seja, cada célula com 1 ha. As coletas foram realizadas com a utilização de um aparelho receptor GPS, resultando em 71 pontos, nas camadas de $0-20 \mathrm{~cm}$ e $20-40 \mathrm{~cm}$ de profundidade. O modelo matemático inserido no software Araquá assume os valores das propriedades físicas e químicas da camada mais profunda $(20-40 \mathrm{~cm})$ do solo para realização dos cálculos até o topo do corpo de água subterrânea. As propriedades físicas e químicas do solo consideradas foram densidade do solo, teor de umidade na capacidade de campo, teor de carbono orgânico e porosidade do solo na zona saturada.

Os parâmetros do herbicida tebuthiuron considerados foram dose da aplicação, coeficiente de sorção normalizado para carbono orgânico, meia-vida no solo e padrão de potabilidade. A dose considerada para o tebuthiuron foi de $1.200 \mathrm{~g} \mathrm{ha}^{-1}$; o coeficiente de sorção normalizado para carbono orgânico $\left(\mathrm{K}_{\mathrm{oc}}\right)$ foi de $80 \mathrm{~mL} \mathrm{~g}^{-1}$; a meia-vida $\left(\mathrm{t}_{1 / 2}\right)$, de 360 dias; e o padrão de potabilidade, de $500 \mathrm{ug} \mathrm{L}^{-1}$ (Moraes et al., 2012). Para a obtenção das estimativas das concentrações do tebuthiuron na água subterrânea, foi utilizado o software Araquá.

Pesq. agropec. bras., Brasília, v.51, n.12, p.1992-1999, dez. 2016 DOI: 10.1590/S0100-204X2016001200010 
Os 71 pontos de solo amostrados foram submetidos aos cálculos com auxílio do software Araquá, em conjunto com os dados climáticos, do corpo de água subterrâneo e das lâminas de irrigação. Foram simulados quatro cenários: 1 , profundidade do lençol freático a $200 \mathrm{~cm}$ da superfície do solo e sem irrigação; 2, profundidade do lençol freático a $200 \mathrm{~cm}$ da superfície do solo e lâmina de irrigação anual de $2.500 \mathrm{~mm}$; 3, profundidade do lençol freático a $100 \mathrm{~cm}$ da superfície do solo e sem irrigação; e 4, profundidade do lençol freático a $100 \mathrm{~cm}$ da superfície do solo e lâmina de irrigação anual de $2.500 \mathrm{~mm}$. Os valores para o nível do lençol freático foram adotados de forma fictícia e constante. De forma fictícia em virtude da ausência de poços de observação, e de forma constante por ser a área relativamente pequena. $\mathrm{O}$ valor de profundidade do lençol freático de $100 \mathrm{~cm}$ foi adotado para considerar um cenário extremamente crítico em avaliação de risco ambiental de defensivos agrícolas. Os dados de precipitação, evapotranspiração e irrigação são considerados como anuais pelo software Araquá.

No software Araquá, utiliza-se a forma generalizada para solos com várias camadas (ou horizontes), e o cálculo matemático da concentração estimada na água subterrânea tem como base o conjunto de equações apresentadas por Rao et al. (1985), AF $=\exp \left(-t_{r} \times k\right)$ em que AF é o fator de atenuação, $t_{r}$ representa o tempo de percurso, e k a constante da taxa de degradação do agrotóxico no solo.

A meia-vida do agrotóxico $\left(t^{1} / 2\right)$ está relacionada com $\mathrm{k}$, conforme a equação $\mathrm{k}=0,693 / \mathrm{t}^{1} / 2$. O tempo de percurso é calculado pela equação, $\mathrm{t}_{\mathrm{r}}=[(\mathrm{L} \times \mathrm{FC}) / \mathrm{q}] \times \mathrm{RF}$, em que $L$ representa a distância até a água subterrânea, FC a capacidade de campo do solo, e q a taxa de recarga hídrica líquida (diferença entre a precipitação, adicionada da irrigação, e a evapotranspiração).

$\mathrm{O}$ fator de retardamento (RF) do movimento do agrotóxico é obtido pela equação $\mathrm{RF}=1+[(\mathrm{BD} \times \mathrm{OC}$ $\left.\times \mathrm{K}_{\mathrm{oc}} \mathrm{g} / \mathrm{FC}\right]$ na qual BD é a densidade do solo, $\mathrm{OC}$ a fração de carbono orgânico do solo, $\mathrm{K}_{\mathrm{oc}} \mathrm{o}$ coeficiente de partição do agrotóxico normalizado para a fração de carbono orgânico.

A massa do defensivo agrícola que chega até o corpo de água subterrânea é calculada conforme equação $\mathrm{M}=\mathrm{D} \times \mathrm{AF}$ em que $\mathrm{D}$ é a dose inicial do defensivo agrícola, e AF o fator de atenuação do defensivo agrícola.
A concentração $(\mathrm{C})$ doagrotóxico naágua subterrânea é estimada pela equação $\mathrm{C} \cong \mathrm{M} /(\mathrm{p} \times \mathrm{d} \times \mathrm{a}) \mathrm{em}$ que $\mathrm{M}$ é a massa prevista do agrotóxico que chega até o lençol freático, $p$ representa a porosidade do aquífero, $\mathrm{d}$ é a profundidade de mistura dentro do corpo de água subterrâneo (assumido como $2 \mathrm{~m}$ ), e a representa a área de 1 hectare $\left(10.000 \mathrm{~m}^{2}\right)$.

$\mathrm{O}$ modelo AF inserido no software Araquá foi validado em estudos desenvolvidos por Matallo et al. (2005) e Martins et al. (2007).

Após a obtenção das estimativas pontuais de concentração do tebuthiuron na água subterrânea, foram calculadas a estatística descritiva dos resultados e a análise geoestatística.

A estatística descritiva foi obtida calculando-se o valor médio, mínimo, máximo, desvio-padrão, variância e coeficiente de variação. Os valores do coeficiente de variação foram comparados com os mesmos critérios utilizados por Silva et al. (2008), como de média variabilidade para os valores entre $12 \%$ e $60 \%$, e os valores abaixo e acima deste intervalo como de baixa e alta variabilidade, respectivamente.

A análise geoestatística foi aplicada com o uso do variograma abaixo, o qual descreve o componente de dependência espacial de uma função aleatória (Oliver \& Webster, 2014).

$$
\gamma(h)=\left(\frac{1}{2 N(h)}\right) \sum_{i=1}^{N(h)}\left[z\left(x_{i}\right)-z\left(x_{i}+h\right)\right]^{2}
$$

em que $\gamma(\mathrm{h})$ representa metade da esperança matemática do quadrado da diferença entre os valores dos pares de concentrações estimadas separados pelo vetor de distância $h$, isto é, a variância; (h) é uma função dependente do ângulo e da distância do vetor $\mathrm{h}$ entre os números de pares de valores das concentrações estimadas $x_{i}+h, x_{i}$; $z$ é o valor dos pares de concentrações estimadas.

Para elaboração e ajuste do variograma, com base nas pressuposições de estacionariedade da hipótese intrínseca, foi utilizada a extensão Geostatistical Analyst do programa ArcGIS 10.1. (Environmental Systems Research Institute, Redlands, CA, EUA).

Para medir a dependência espacial entre as concentrações estimadas, foi calculado o valor para o índice de dependência espacial, IDE $=\left[\mathrm{C} /\left(\mathrm{C}+\mathrm{C}_{0}\right)\right] \times 100$, conforme utilizado por Lima et al. (2009), em que IDE é o índice de dependência espacial, $\mathrm{C}$ é a componente 
estrutural, e $\mathrm{C}_{0}$ o efeito pepita. Valores $\leq 25 \%$ indicam fraca dependência espacial; entre $25 \%$ e $75 \%$ indicam moderada dependência espacial, e $\geq 75 \%$, forte dependência espacial.

Adicionalmente, foi calculado o coeficiente de correlação de Pearson entre a concentração estimada de tebuthiuron na água subterrânea e as propriedades físicas e químicas do solo, considerando as condições de irrigação e as profundidades do lençol freático de $100 \mathrm{~cm}$ e $200 \mathrm{~cm}$.

O processo de interpolação dos dados foi realizado após análise do variograma, considerando duas situações: se o valor do IDE fosse $\leq 25 \%$ ou o variograma apresentasse efeito pepita puro, seria aplicado o processo de interpolação pelo inverso da distância; caso contrário, seria aplicada a krigagem ordinária.

O método do inverso da distância,

$$
\mathrm{iv}=\sum_{\mathrm{i}-1}^{\mathrm{n}}\left(\frac{1}{\mathrm{~d}_{1}^{\mathrm{p}}}\right) \times \mathrm{v}_{\mathrm{i}} / \sum_{\mathrm{i}=1}^{\mathrm{n}}\left(\frac{1}{\mathrm{~d}_{1}^{\mathrm{p}}}\right),
$$

estima valores para locais não amostrados como uma média dos valores dos dados dentro de uma vizinhança. Dessa forma, o cálculo da média é ponderado pelo inverso da distância (Yasreb et al., 2009), em que $\mathrm{v}_{\mathrm{i}}$ é o valor amostrado; $\mathrm{d}_{1}$ é a distância dos locais das amostras ao ponto estimado; e p é uma potência da distância.

A krigagem ordinária utiliza um estimador linear não viciado com mínima variância (BLUEBest Linear Unbiased Estimator) para interpolação do atributo medido em posições não amostradas. Linear porque suas estimativas são calculadas por combinações lineares; unbiased (sem viés) porque o erro de estimativa esperado é nulo; e best porque seu objetivo é minimizar a variância destes erros de estimativa. O estimador é uma combinação linear que é uma média móvel e leva em conta a estrutura de variabilidade encontrada para aquela variável (medida), expressa pelo variograma e pela localização dos valores conhecidos. Pontos próximos da posição a ser interpolada apresentam maiores pesos que os mais distantes.

A krigagem ordinária,

$$
\mathrm{Z}\left(\mathrm{x}_{0}\right)=\sum_{\mathrm{i}=1}^{\mathrm{n}} \lambda_{\mathrm{i}} \times \mathrm{Z}\left(\mathrm{x}_{\mathrm{i}}\right)
$$

em que $\mathrm{Z}\left(\mathrm{x}_{0}\right)$ é o valor estimado para local $\mathrm{x}_{0}$ não amostrado; $\mathrm{Z}\left(\mathrm{x}_{\mathrm{i}}\right)$ são os valores obtidos por amostragem no campo; e $\lambda_{\mathrm{i}}$ são os pesos associados ao valor medido na posição $\mathrm{x}_{\mathrm{i}}$.

\section{Resultados e Discussão}

Considerando as lâminas de irrigação anuais e profundidades do lençol freático, os valores obtidos das concentrações estimadas para cada lâmina de irrigação simulada foram relativamente baixos, demonstrando conformidade com o padrão de potabilidade para o tebuthiuron $\left(\leq 500 \mathrm{ug} \mathrm{L^{-1 }}\right)$. Como esperado, conforme simulou-se a lâmina de irrigação, as concentrações estimadas do tebuthiuron na água subterrânea aumentaram. Essa situação é caracterizada por uma maior taxa de recarga hídrica, promovendo uma maior percolação da água no solo e, consequentemente, uma maior lixiviação do tebuthiuron até o topo do lençol freático (Tabela 1). Resultados obtidos por Zhang et al. (2013) demonstraram alta concentração de defensivos agrícolas na água subterrânea em áreas de cultivo irrigado.

Conforme a profundidade do lençol freático simulada diminuiu, as concentrações estimadas do tebuthiuron na água subterrânea aumentaram. Isso se deve à redução do percurso do deslocamento do tebuthiuron no perfil do solo até o topo do lençol freático e, também, ao fato de o tebuthiuron ser uma molécula persistente, com longa meia-vida. Di Guardo \& Finizio (2015) demostraram que áreas com corpos

Tabela 1. Análise estatística das concentrações estimadas de tebuthiuron na água subterrânea, em área de cultivo de cana-de-açúcar, no Município de São Manuel, SP, considerando a profundidade do lençol freático a $100 \mathrm{~cm}$ e a $200 \mathrm{~cm}$ da superfície do solo.

\begin{tabular}{lcccccc}
\hline $\begin{array}{l}\text { Lâmina simulada } \\
(\mathrm{mm})\end{array}$ & $\begin{array}{c}\text { Média } \\
\left(\mathrm{ug} \mathrm{L}^{-1}\right)\end{array}$ & $\begin{array}{c}\text { Mínimo Máximo } \\
\left.(\mathrm{ug} \mathrm{L})^{-1}\right)\end{array}$ & $\left.(\mathrm{ug} \mathrm{L})^{-1}\right)$ & & Variância & $\begin{array}{c}\text { CV } \\
(\%)\end{array}$ \\
\hline \multicolumn{7}{c}{ Profundidade do lençol freático a $100 \mathrm{~cm}$} \\
0 & 64,97 & 22,46 & 121,58 & 20,43 & 417,38 & 31,44 \\
2.500 & 154,15 & 117,67 & 223,54 & 18,49 & 341,88 & 12,00 \\
\hline \multicolumn{7}{c}{ Profundidade do lençol freático a 200 cm } \\
0 & 26,18 & 3,23 & 67,10 & 14,25 & 203,06 & 54,43 \\
2.500 & 134,49 & 90,07 & 201,19 & 19,58 & 383,37 & 14,55 \\
\hline \multicolumn{7}{c}{}
\end{tabular}

DP, desvio-padrão; CV, coeficiente de variação.

Pesq. agropec. bras., Brasília, v.51, n.12, p.1992-1999, dez. 2016 DOI: 10.1590/S0100-204X2016001200010 
de água subterrâneos mais próximos à superfície do solo são mais vulneráveis à percolação de defensivos.

Em relação ao coeficiente de variação, todas as simulações indicaram média variabilidade (Tabela 1). No entanto, nas simulações que consideraram a lâmina de irrigação, os valores $(<12 \%)$ estão próximos da baixa variabilidade (Silva et al., 2008), podendo afetar o comportamento do variograma. Isso porque a baixa variabilidade dos dados é um indício de que a área de estudo possui uma grande parcela de homogeneidade, podendo causar o chamado efeito pepita puro.

Para a profundidade do lençol freático a $100 \mathrm{~cm}$ da superfície do solo, os variogramas apresentaram fraca dependência espacial (Tabela 2). Isso ocorre pelo fato de o valor do efeito pepita estar próximo ao patamar já na origem $(\mathrm{h}=0 \mathrm{~m})$. $\mathrm{O}$ variograma referente à lâmina de irrigação de $2.500 \mathrm{~mm}$ apresentou efeito

Tabela 2. Parâmetros e modelo de ajuste dos variogramas para concentrações do tebuthiuron, em área de cultivo de cana-de-açúcar, no Município de São Manuel, SP, considerando a profundidade do lençol freático a $100 \mathrm{~cm}$ e a $200 \mathrm{~cm}$ da superfície do solo.

\begin{tabular}{llcccc}
\hline $\begin{array}{l}\text { Lâmina simulada } \\
(\mathrm{mm})\end{array}$ & Modelo & $\mathrm{A}_{0}$ & $\mathrm{C}_{0}$ & $\mathrm{C}$ & $\begin{array}{c}\text { IDE } \\
(\%)\end{array}$ \\
\hline \multicolumn{5}{c}{$(\mathrm{m})$} \\
0 & Profundidade do lençol freático a $100 \mathrm{~cm}$ \\
2.500 & Esférico & 348,84 & 388,82 & 78,41 & 16,78 \\
\hline \multicolumn{5}{c}{ Profundidade do lençol freático a $200 \mathrm{~cm}$} \\
0 & Esférico & - & 371,93 & 0 & 0 \\
2.500 & Esférico & 372,02 & 165,22 & 63,75 & 27,83 \\
\hline
\end{tabular}

$\mathrm{A}_{0}$, valor do alcance obtido; $\mathrm{C}_{0}$, efeito pepita; $\mathrm{C}$, componente estrutural; IDE, índice de dependência espacial. pepita puro, caracterizando ausência de dependência espacial (IDE $=0$ ). Tal fato pode ser explicado pela baixa variabilidade dos dados na área, causando independência espacial destes. Apesar de apresentar uma pequena estrutura, o variograma no qual não houve a aplicação de lâmina de irrigação pode ser classificado como uma estrutura de efeito pepita puro.

As mesmas situações ocorreram com os variogramas referentes à profundidade do lençol freático de $200 \mathrm{~cm}$. Apesar de o variograma que não considerou a aplicação de lâmina de irrigação mostrar uma dependência espacial moderada, a estrutura dele está próxima de apresentar efeito pepita puro.

Em vista dos resultados apresentados pelos variogramas, o método de interpolação escolhido para obtenção dos mapas temáticos foi o inverso da distância, uma vez que os dados não apresentaram dependência espacial suficiente para aplicação da krigagem ordinária. Quando há a impossibilidade de obtenção de um modelo de correlação espacial, devem-se considerar interpoladores não estocásticos (Yamamoto \& Landim, 2013).

Em relação às profundidades do lençol freático de $100 \mathrm{~cm}$ e $200 \mathrm{~cm}$, nota-se que a correlação entre a concentração estimada do tebuthiuron na água subterrânea e o teor de carbono orgânico no solo (Tabela 3), para as duas camadas do solo, ocorre de forma acentuada e inversamente proporcional quando não há aplicação da lâmina de irrigação (Figura $1 \mathrm{Aa}$, $\mathrm{Ba}$ ). No entanto, quando há irrigação, a correlação passa a ser acentuada e diretamente proporcional à densidade do solo na camada de 20 a $40 \mathrm{~cm}$, e inversamente proporcional à porosidade do solo na zona saturada (Figura $1 \mathrm{Ab}, \mathrm{Bb}$ ).

Tabela 3. Coeficiente de Correlação de Pearson entre a concentração estimada de tebuthiuron na água subterrânea e as propriedades do solo, considerando a profundidade do lençol freático a $100 \mathrm{~cm}$ e $200 \mathrm{~cm}$ da superfície do solo, em área de cana-de-açúcar, São Manuel, SP.

\begin{tabular}{|c|c|c|c|c|c|c|c|}
\hline \multirow{2}{*}{$\begin{array}{l}\text { Lâmina simulada } \\
(\mathrm{mm})\end{array}$} & \multicolumn{2}{|c|}{ Capacidade de campo (CC) } & \multicolumn{2}{|c|}{ Densidade do solo (DS) } & \multicolumn{2}{|c|}{ Carbono orgânico (CO) } & \multirow[t]{2}{*}{ POR } \\
\hline & $(0-20 \mathrm{~cm})$ & $(20-40 \mathrm{~cm})$ & $(0-20 \mathrm{~cm})$ & $(20-40 \mathrm{~cm})$ & $(0-20 \mathrm{~cm})$ & $(20-40 \mathrm{~cm})$ & \\
\hline & \multicolumn{7}{|c|}{ Profundidade do lençol freático a $100 \mathrm{~cm}$} \\
\hline 0 & $-0,34^{*}$ & $-0,54^{*}$ & $0,36^{*}$ & $0,61^{*}$ & $-0,82 *$ & $-0,88^{*}$ & $-0,64 *$ \\
\hline \multirow[t]{2}{*}{2.500} & $-0,32 *$ & $-0,58^{*}$ & $0,33 *$ & $0,86^{*}$ & $-0,54 *$ & $-0,59 *$ & $-0,94 *$ \\
\hline & \multicolumn{7}{|c|}{ Profundidade do lençol freático a $200 \mathrm{~cm}$} \\
\hline 0 & $-0,34^{*}$ & $-0,50 *$ & $0,36^{*}$ & $0,54^{*}$ & $-0,81^{*}$ & $-0,88^{*}$ & $-0,55^{*}$ \\
\hline 2.500 & $-0,34^{*}$ & $-0,60 *$ & $0,35^{*}$ & $0,81^{*}$ & $-0,65^{*}$ & $-0,72 *$ & $-0,87^{*}$ \\
\hline
\end{tabular}

POR, porosidade do solo na zona saturada. *Significativo a $5 \%$ de probabilidade. 
Observa-se então que, com recarga hídrica baixa em virtude da ausência de aplicação de lâmina de irrigação, a concentração estimada de tebuthiuron na água subterrânea está mais correlacionada ao teor de carbono orgânico no solo. E, por ser inversamente proporcional, quanto maior o teor de carbono orgânico no solo, menor a concentração de tebuthiuron na água subterrânea e vice-versa. Áreas com menor teor de carbono orgânico caracterizam uma alta mobilidade da molécula do defensivo agrícola no solo, uma vez que a possibilidade de ela ser adsorvida é reduzida (Fenoll et al., 2014).
Porém, quando há uma aplicação de lâmina de irrigação, a concentração estimada de tebuthiuron na água subterrânea mostra-se mais correlacionada à densidade do solo na camada de 20 a $40 \mathrm{~cm}$ e à porosidade do solo na zona saturada. Maior saturação do solo permite diversas reações químicas, tais como biodegradação, filtragem e difusão (Muhammad et al., 2015). Tal situação promove a menor concentração de tebuthiuron na água subterrânea. Menor porosidade na zona saturada do solo indica um menor volume de água para diluir a massa do tebuthiuron que chega ao lençol freático, além de diminuir processos químicos, o que resulta em uma maior concentração.
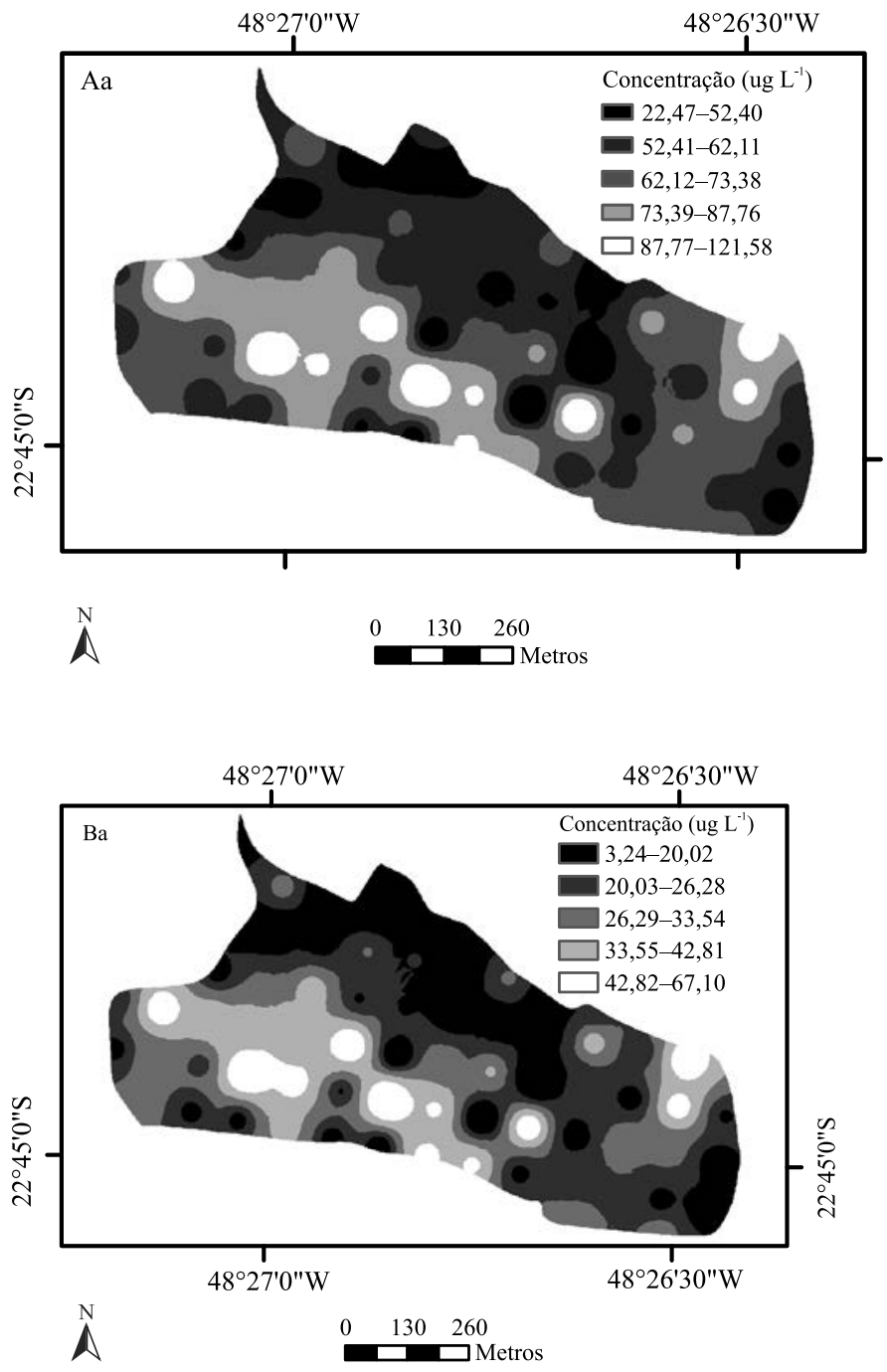
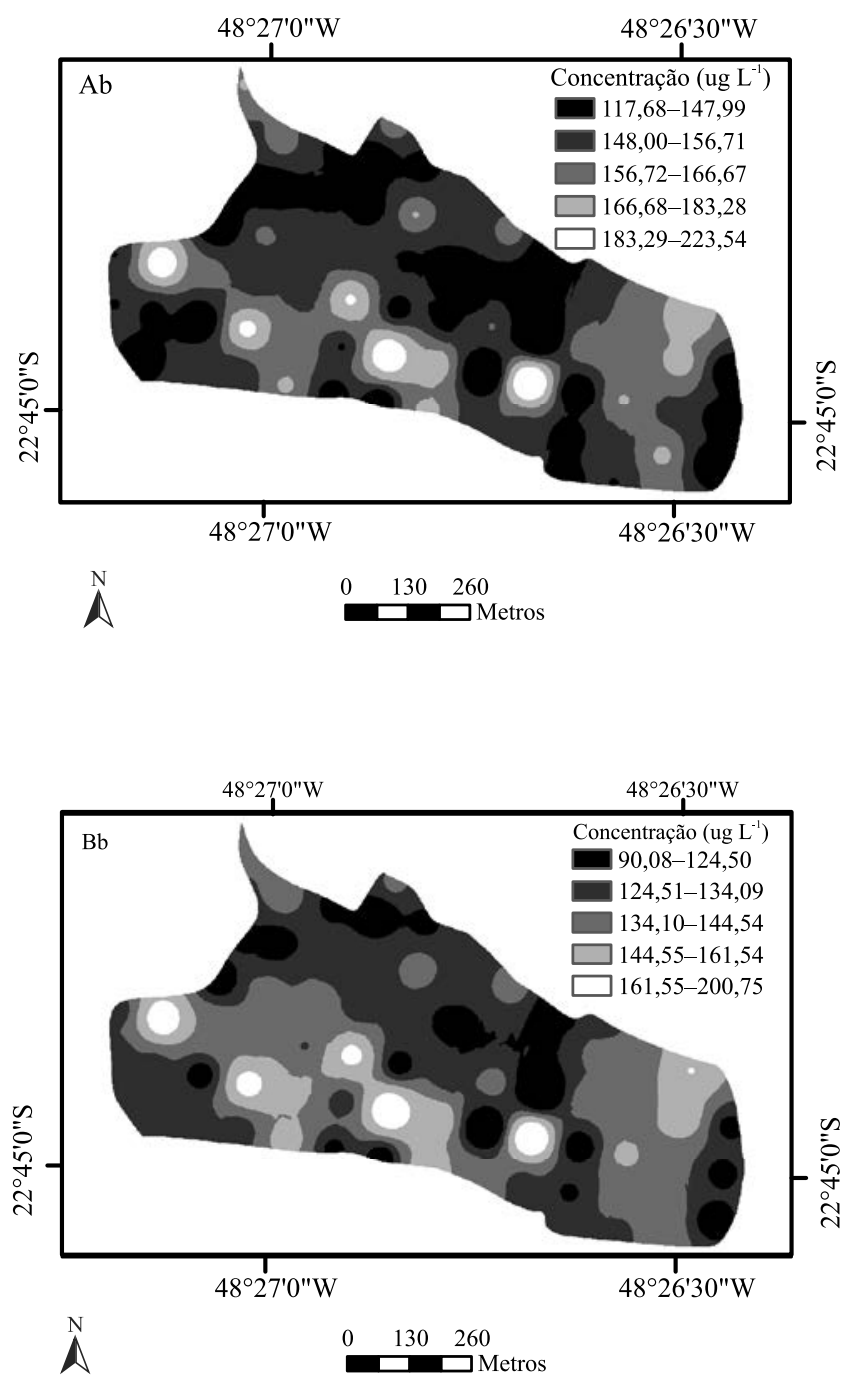

Figura 1. Concentrações estimadas de tebuthiuron na água subterrânea, a $100 \mathrm{~cm}$ (A) e a $200 \mathrm{~cm}$ (B), considerando uma lâmina de irrigação de 0 mm (a) e 2.500 mm (b), em área de cultivo de cana-de-açúcar, no Município de São Manuel, SP. 


\section{Conclusões}

1. Para as profundidades do lençol freático de 100 e $200 \mathrm{~cm}$ sem e com lâmina de irrigação anual de $2.500 \mathrm{~mm}$, não há concentrações estimadas de tebuthiuron acima do valor padrão de potabilidade.

2. A interpolação das estimativas das concentrações de tebuthiuron na água subterrânea proporciona, de forma rápida, a visualização das áreas com maior risco de contaminação, bem como as variáveis do solo mais correlacionadas com as concentrações estimadas.

\section{Agradecimentos}

À Coordenação de Aperfeiçoamento de Pessoal de Nível Superior (Capes, PDSE no 99999.002573/201408), pelo apoio financeiro.

\section{Referências}

AGRITEMPO: Sistema de Monitoramento Agrometeorológico. Estatísticas. 2014. Disponível em: <http://www.agritempo.gov. br/agritempo/index.jsp>. Acesso em: 1 ago. 2014.

AKBAR, T.A.; LIN, H. GIS based ArcPRZM-3 model for bentazon leaching towards groundwater. Journal of Environmental Sciences, v.22, p.1854-1859, 2010.

CERDEIRA, A.L.; PESSOA, M.C.P.Y.; SANTOS, N.P.G. dos; LANCHOTE, V.L. Lixiviação de atrazina em solo em área de recarga do Aquífero Guarani. Revista Brasileira de Herbicidas, v.4, p.92-101, 2005. DOI: 10.7824/rbh.v4i2.29.

CIIAGRO: Centro Integrado de Informações Agrometeorológicas. Monitoramento agrometeorológico e climático: CIIAGRO Online. 2014. Disponível em: <http://www.ciiagro.sp.gov.br/>. Acesso em: 1 ago. 2014.

DI GUARDO, A.; FINIZIO, A. A client-server software for the identification of groundwater vulnerability to pesticides at regional level. Science of the Total Environment, v.530/531, p.247-256, 2015. DOI: 10.1016/j.scitotenv.2015.05.112.

CLAESSEN, M.E.C. (Org.). Manual de métodos de análise de solo. 2.ed. rev. e atual. Rio de Janeiro: EMBRAPA-CNPS, 1997. 212p. (EMBRAPA-CNPS. Documentos, 1).

ESTADOS UNIDOS. National Library of Medicine. Hazardous Substances Data Bank (HSDB). Disponível em: <https://toxnet. nlm.nih.gov/cgi-bin/sis/htmlgen?HSDB>. Acesso em: $30 \mathrm{jul}$. 2013.

FENOLL, J.; VELA, N.; NAVARRO, G.; PÉREZ-LUCAS, G.; NAVARRO, S. Assessment of agro-industrial and composted organic wastes for reducing the potential leaching of triazine herbicide residues through the soil. Science of the Total Environment, v.493, p.124-132, 2014. DOI: 10.1016/j. scitotenv.2014.05.098
FRANCO-BERNARDES, M.F.; MASCHIO, L.R.; AZEREDOOLIVEIRA, M.T.V. de; ALMEIDA, E.A. de. Biochemical and genotoxic effects of a commercial formulation of the herbicide tebuthiuron in Oreochromis niloticus of different sizes. Ecotoxicology and Environmental Contamination, v.9, p.59-67, 2014. DOI: 10.5132/eec.2014.01.008.

GIANNOULI, D.D.; ANTONOPOULOS, V.Z. Evaluation of two pesticide leaching models in an irrigated field cropped with corn. Journal of Environmental Management, v.150, p.508-515, 2015. DOI: 10.1016/j.jenvman.2014.12.044.

LIMA, J.S. de S.; SATTLER, M.A.; PASSOS, R.R.; OLIVEIRA II, P.C.; SOUZA, G.S. de. Variabilidade espacial de atributos físicos de um argissolo vermelho-amarelo sob pastagem e vegetação secundária em regeneração natural. Engenharia Agrícola, v.29, p.185-195, 2009. DOI: 10.1590/S0100-69162009000200002.

MARTINS, E.L.; WEBER, O.L.S.; DORES, E.F.G.C.; SPADOTTO, C.A. Leaching of seven pesticides currently used in cotton crop in Mato Grosso State - Brazil. Journal of Environmental Science and Health, Part B, v.42, p.877-882, 2007. DOI: $10.1080 / 03601230701623217$.

MATALLO, M.B.; SPADOTTO, C.A.; LUCHINI, L.C.; GOMES, M.A.F. Sorption, degradation, and leaching of tebuthiuron and diuron in soil columns. Journal of Environmental Science and Health, v.40, p.39-43, 2005.

MORAES, D.A. de C.; ZIMBACK, C.R.L.; SPADOTTO, C.A. Espacialização das estimativas de contaminação de águas subterrâneas por tebuthiuron. Irriga, v.17, 2012. DOI: 10.15809/ irriga.2012v17n3p299.

MUHAMMAD, A.M.; ZHONGHUA, T.; DAWOOD, A.S.; EARL, B. Evaluation of local groundwater vulnerability based on DRASTIC index method in Lahore, Pakistan. Geofísica Internacional, v.54, p.67-81, 2015. DOI: 10.1016/j.gi.2015.04.003.

NOFZIGER, D.L.; HORNSBY, A.G. Chemical Movement in Layered Soils: user's manual. Gainesville: University of Florida, 1987. 44p. (Circular, 780).

OLIVER, M.A.; WEBSTER, R. A tutorial guide to geostatistics: computing and modelling variograms and kriging. Catena, v.113, p.56-69, 2014.

PULLAN, S.P.; WHELAN, M.J.; RETTINO, J.; FILBY, K.; EYRE, S.; HOLMAN, I.P. Development and application of a catchment scale pesticide fate and transport model for use in drinking water risk assessment. Science of the Total Environment, v.563/564, p.434-447, 2016.

RAO, P.S.C.; HORNSBY, A.G.; JESSUP, R.E. Indices for ranking the potential for pesticide contamination of groundwater. Soil and Crop Science Society of Florida, v.44, p.1-8, 1985.

SCORZA JÚNIOR, R.P.; SILVA, J.P. da. Potencial de contaminação da água subterrânea por pesticidas na bacia do Rio Dourados, MS. Pesticidas: Revista de Ecotoxicologia e Meio Ambiente, v.17, p.87-106, 2008. DOI: 10.5380/pes.v17i0.10666.

SILVA, P.V. da; MONQUERO, P.A.; MUNHOZ, W.S. Controle em pós-emergência de plantas daninhas por herbicidas utilizados na cultura da cana-de-açúcar. Revista Caatinga, v.28, p.21-32, 2015. 
SILVA, S. de A.; LIMA, J.S. de S.; SOUZA, G.S. de; OLIVEIRA, R.B. de. Avaliação de interpoladores estatísticos e determinísticos na estimativa de atributos do solo em agricultura de precisão. Idesia, v.26, p.75-81, 2008. DOI: 10.4067/S071834292008000200010 .

SILVEIRA, J.L.; MARTINELLI, V.J.; VANE, L.F.; FREIRE JUNIOR, J.C.; VIGOUROUX, R.A.Z.; TUNA, C.E.; LAMAS, W. de Q.; PAULINO, R.F.S. Incorporation of hydrogen production process in a sugar cane industry: steam reforming of ethanol. Applied Thermal Engineering, v.71, p.94-103, 2014.

SPADOTTO, C.A.; MORAES, D.A. de C.; BALLARIN, A.W.; LAPERUTA FILHO, J.; COLENCI, R.A. ARAquá: software para avaliação de risco ambiental de agrotóxico. Campinas: Embrapa Monitoramento por Satélite, 2010. (Embrapa Monitoramento por Satélite. Boletim de pesquisa e desenvolvimento, 7). Disponível em: <http://ainfo.cnptia.embrapa.br/digital/bitstream/ item/35294/1/boletim6ATUAL.pdf $>$. Acesso em: 31 ago. 2014.

TIKTAK, A.; BERG, F. van den; BOESTEN, J.J.T.I.; KRAALINGEN, D. van; LEISTRA, M.; LINDE, A.M.A. van der.
Manual of FOCUS PEARL version 1.1.1. Bilthoven: RIVM/ Alterra, 2000.

TOFOLI, G.R.; VELINI, E.D.; NEGRISOLI; E.; CAVENAGHI, A.L.; MARTINS, D. Dinâmica do tebuthiuron em palha de canade-açúcar. Planta Daninha, v.27, 2009. DOI: 10.1590/S010083582009000400020 .

YAMAMOTO, J.K.; LANDIM, P.M.B. Geoestatística: conceitos e aplicações. São Paulo: Oficina de Textos, 2013. 215p.

YASREBI, J.; SAFFARI, M.; FATHI, H.; KARIMIAN, N.; MOAZALLAHI, M.; GAZNI, R. Evaluation and Comparison of Ordinary Kriging and Inverse Distance Weighting Methods for Prediction of Spatial Variability of Some Soil Chemical Parameters. Research Journal of Biological Sciences, v.4, p.93$102,2009$.

ZHANG, C.; LIAO, X.; LI, J.; XU, J.; LIU, M.; DU, B.; WANG, $\mathrm{Y}$. Influence of long-term sewage irrigation on the distribution of organochlorine pesticides in soil-groundwater systems. Chemosphere, v.92, p.337-343, 2013.

Recebido em 15 de abril de 2015 e aprovado em 25 de julho de 2016 